\title{
Mental status examination of borderline personality disorder
}

\begin{abstract}
The study aimed to identify characteristics of Borderline Personality Disorder (BPD) patients using case study method and mental status examination (MSE). Five cases of BPD (3 males, 2 females; aged 17-23years) identified using semi-structured interview schedule including case history, MSE and DSM-IV-TR criteria were taken from a Psychiatric Centre in India. The themes and characteristics were identified from in-depth clinical interviews and direct observation of the patients. Evaluation of the emotional and cognitive functioning was done. General appearance of patients was appropriate. Males appeared dull, sad and reclusive while females were elated. Psychomotor activity was slow, increased in some cases. Speech was slow; they spoke in muffled voices and sometimes were loud. Emotional state indicated feelings of anger, hurt, boredom, and depression. Affect was not compatible with the idea and content of thoughts and situations. Thought processes involved loose associations, fears and somatic trends in males. Suicidal ideation was common in all patients. Sensorium and mental capacity revealed that their remote memory was adequate, recent memory was impaired while immediate memory was poor. They had poor attention and concentration with average general awareness. Insight and judgement capacity showed that patients were aware of their mental problem. They wanted their problems to be shared, heard and understood. A characteristic profile for evaluating emotional and cognitive functioning of BPD was developed in the study using case study.
\end{abstract}

Keywords: borderline personality disorder, mental status examination, characteristic features
Volume 8 Issue 2 - 2017

Shalini Choudhary

Department of Psychology, University of Allahabad, India

Correspondence: Shalini Choudhary, Research Scholar, Department of Psychology, University of Allahabad, Allahabad, 211002, India, Email shalini.301 I@gmail.com

Received: March 23, 2017 | Published: September 15, 2017

\section{Mental status examination of borderline personality disorder}

Borderline Personality Disorder (BPD) is a serious personality disorder. People with BPD show a pattern of behavior characteristics marked by impulsivity and instability in interpersonal relationships, self-image, and moods. BPD is a debilitating disorder that occurs in approximately $1-4 \%$ of the general population and about $75 \%$ are females. ${ }^{1-3}$ Recent studies have found that BPD is actually more prevalent affecting almost $5.9 \%$ of the population; and there was no difference found among the rates of BPD among women vs. men. ${ }^{4}$ Within a sample of personality-disordered patients, Zanarini et al., ${ }^{5}$ found gender differences in the "type of disorder of impulse in which they specialized", with male borderline patients significantly more likely to meet criteria for lifetime substance use and females significantly more likely to meet criteria for life-time eating disorders. Common clinical features of BPD are frequent intense mood swings, the inability to be alone nor to tolerate intimacy, fears of abandonment, extreme dependency on others alternating with sudden hostility, perceiving others as all good or all bad ("splitting"), chronic self-mutilation (often described as relieving emotional pain), and chronic suicidality. Suicide attempts serve to punish others, escape suffering, and to communicate distress. ${ }^{6,7}$ These patients are argumentative, irritable, sarcastic, quick to take offence and very hard to live with. Self-mutilation is one of the most discriminating signs of BPD. ${ }^{8}$ Self-injurious behavior is associated with relief from anxiety or dysphoria and with analgesia (absence of the experience of pain in the presence of a theoretically painful stimulus). ${ }^{8}$ Other researchers have emphasized interpersonal problems and conflict as important precipitating events to the parasuicide. ${ }^{9-12}$ According to the Diagnostic and Statistical Manual of Mental Disorders, fourth edition, Text Revision (DSM-IV-TR), ${ }^{13,14}$ these conditions are defined by maladaptive personality characteristics beginning early in life (early adulthood) that have consistent and serious effects on functioning. BPD is frequently seen in clinical practice, characterized by emotional turmoil and chronic suicidality (suicide ideation and attempts). Several studies stress the role of impulsiveness as a core construct of BPD. ${ }^{15-17}$ Emotion dysregulation is supposed to be a central mechanism of the disorder. ${ }^{6}$ Patients with BPD are supposed to show high sensitivity and intense reactions to emotional stimuli as well as longer latencies to return to their baseline emotional state. ${ }^{18}$ BPD commonly co-occurs with a variety of Axis I disorder ranging from mood \& anxiety disorders (esp. panic \& Post Traumatic Stress Disorder), to substance use \& eating disorders. ${ }^{19}$ Several studies have shown high comorbidity between BPD and substance use disorders (SUD). ${ }^{20-22}$ It has been suggested that the comorbidity occurs because both conditions share the common etiologies ${ }^{23}$ and are part of the same domain of psychopathology (i.e., impulse control disorders, ${ }^{24,25}$ Many authors consider substance use a manifestation of impulsivity, which is seen as a core feature of the disorder. ${ }^{26,27} \mathrm{BPD}$ is frequently associated with depression, domestic violence, and suicide. ${ }^{6-29}$ Family and marital relationships are frequently disrupted, and health care utilization is excessive. ${ }^{30,31}$ There are many diagnostic controversies in the identification of borderline patients which include instruments and diagnostic processes used in assessment of BPD. Various methods to assess BPD are being followed. Case history and diagnostic measures like self report inventories and questionnaires are popularly being used around the world in all cultures. History taking and direct observation using clinical interview is the strongest tool to assess psychiatric patients. Direct observation of the patient is strongly recommended in clinical settings. Psychiatric observation is dome by evaluating the Mental Status Examination (MSE). The MSE is an assessment of the patient's current state of mind. It assesses the range, quality, and depth of perception, thought processes, feelings, and psychomotor activities. Direct observation of the patient is required for the completion of the MSE. This observation occurs before, during and after the formal 
clinical interview while the clinician is in direct view of the patient. Specific questions to assess certain domains of the MSE are also used. Despite the attention it receives in the west, there are hardly few studies on eastern populations. Eastern Perspective explains the disorder in terms of family and society. Very few studies on BPD have been done in the Indian context. Findings of a study suggest that Paris $^{32}$ used a case, a patient of Indian descent who developed BPD after she immigrated to Canada, to elaborate the hypothesis that BPD appears to be highly sensitive to the socio-cultural context. He insisted that risk factors underlying BPD exist in developing countries and that some traditional cultures as India provide protective factors that suppress the overt expression of BPD symptoms. BPD in India exists and may be under diagnosed in the clinical setting. These patients had a high incidence of childhood sexual/physical abuse, comorbid depression and substance abuse, frequent and severe self-injurious behavior, and a high risk for suicide. It emphasized that clinicians in India need to be more aware of the possibility of BPD in their patients, particularly those who are frequent self-abusers. ${ }^{33}$ Another study aimed to identify the type of personality disorder commonly associated with deliberate self harm and found that the commonest disorder was emotionally unstable (both borderline and impulsive type) in young people. This was more common in females than in males. ${ }^{34}$ Depressive symptoms were observed in all the cases at the time of admission but were transient..$^{35}$ Eastern studies on BPD are not sufficient and very few studies have done using qualitative methods. One such study attempted at identifying characteristic profile of BPD patients in North India using case history method and MSE. ${ }^{36}$ General purpose of this study was to examine specific features in BPD patients and to use clinical methods such as case history and mental status examination at identifying the characteristics of BPD patients in India. The present study attempted to assess a direct and indirect, subjective and objective observation and formal testing of the client's mental, emotional and behavioral functioning through MSE.

\section{Method}

\section{Participants}

Potential patient participants were considered for entry into the study when they were suspected to have BPD features by the psychiatrists and clinical psychologists. Further investigation was done by the researcher to include the patient as a BPD case. Co morbid patients were also included in the sample due to lack of availability of so-called "pure" cases in various centres. Twenty patients were screened, but only eight cases were found to have BPD features qualifying the criteria of being diagnosed as BPD. Only five cases ( 3 males \& 2 females) completed the in-depth interview. All eligible participants gave their written informed consent according to the guidelines of Institutional Ethics Regulatory Board of University of Allahabad, Allahabad. BPD cases were taken from the Nur Manzil Psychiatric Centre, Lucknow, India. The patients belonged to the places in and around Lucknow city like Unnao, Mau, Rae Barreily and Pratapgarh, Uttar Pradesh, India. Their ages ranged from 17 to 23years.

\section{Clinical techniques}

Case History Method: In-depth clinical interviews were conducted by taking patients' personal history, family history, psychiatric history, information about his social relationships, feelings, experiences, schooling and personality, attitudes and character. Mental Status Examination (MSE): Observation and assessment of the patients' behavior, cognitive and emotional functioning was also done in detail. A Semi-structured Interview Schedule was prepared using the above mentioned clinical techniques. Clinical Interview including case history, DSM-IV-TR criteria, four broader BPD features that encompass these criteria (Domains of Pathology) and MSE was conducted according to the clinical techniques selected for the detailed information of the BPD patient. Informed Consent Form (ICF) was also developed both in Hindi and English in order to inform the patients about the nature of research and to seek their consent for participation in this research.

Procedure: Patients, accessed from the psychiatric centre, were interviewed using semi structured interview schedule. An evaluation of the patient included a detailed psychiatric and social history, demographic variables and clinical features. Detailed information through case history method was obtained. Informants', doctor's and nurses' reports about those patients were also obtained. Hospital records of the inpatients and prescription files of the outpatients were also accessed. Themes were analyzed using, DSM-IV-TR Criteria and MSE. Specific features and commonly found defining characteristics of BPD were identified from the detailed case history and MSE. Accessing BPD patients and administering the complete assessment procedures was very difficult due to various reasons such as:

a. Lack of support from the family as they prohibited the patients from giving the interview

b. Drop-out rate was high as emotional instability is a defining characteristic of these patients

c. Lack of cooperation from the psychiatric centres or clinics.

d. Permission for data collection was not granted in most of the psychiatric centres or clinics

e. Lack of awareness about the disorder among psychiatrists, counsellors and patients themselves

f. Considering these factors, data collection was limited to 5 patients only.

\section{Data analysis}

The tape recorded interview data were transcribed. The abundant data were reduced by following two levels of analysis. Data of each patient was analyzed individually by identifying themes, sub themes and major responses characterizing the themes. Then information of all the clients were grouped together or clubbed according to the themes, sub themes and responses. Table 1 shows MSE of clients revealing significant information about the clients and their emotional and cognitive functioning.

I. General appearance of participants was appropriate and they were well kempt. Males appeared dull, sad and seclusive while females were elated and were cooperative. Psychomotor activity showed slowness and was, increased in some cases.

II. Speech of all clients was slow; they spoke in muffled voices and sometimes were loud.

III. Emotional state of clients was marked by feelings of anger, hurt, boredom, and depression. Male clients showed unstable feelings of sadness, guilt, remorse and loneliness. Females showed intense and labile emotions like elation, excitement irritability, anger, fear and distrust. Affect was not compatible with the idea and content of thoughts and situations.

IV. Thought processes involved loose associations and preoccupations, fears and somatic trends in male clients. Suicidal ideation was common in all clients. 
V. Sensorium and mental capacity of clients revealed that their remote memory was adequate, recent memory was impaired while immediate memory was poor. They had poor attention and concentration as well as average general awareness, calculations and reasoning ability.

VI. Insight and judgment capacity of clients showed that they were aware of their mental problem (which was not severe) and that they caused trouble to others. They wanted their problems to be shared, heard and understood by others. They were uncertain about their plans and goals and were dependent on family members.

\section{Interpretation}

Borderline Personality Disorder (BPD) has been considered a mental health problem that results in terms of human suffering and

Table I Summary of information elicited in MSE psychiatric expenses among adult patients. Personality is an important medium within which culture attempts to ensure social coordination among individuals within the culture and produce individuals who will fulfill social roles. A personality disorder is an enduring pattern of inner experience and behavior that deviates markedly from the expectations of the individual's culture. This study attempted to delineate characteristics of borderline personality disorder in Indian social and cultural context. It was also explored that how an individual's sense of what is real is shaped and structured in his social environment. Apart from the common and unique features, BPD can also have characteristic emotional and cognitive features. The mental status examination (MSE) involved a direct observation of BPD patients and provided important information about the features of BPD (Table 1). Although the data are based on the MSE of five patients ( 3 males and 2 females) only, it provided evidence of gender differences.

\begin{tabular}{|c|c|c|c|}
\hline S.No & Major Sections of Investigation & Sub Sections & Information Obtained \\
\hline \multirow{3}{*}{ I. } & \multirow{3}{*}{$\begin{array}{l}\text { General appearance, behavior and } \\
\text { attitude }\end{array}$} & Age \& grooming & $\begin{array}{l}\text { All participants looked appropriate to their ages and were well } \\
\text { dressed and kempt }\end{array}$ \\
\hline & & $\begin{array}{l}\text { Facial expressions, manners and } \\
\text { attitude }\end{array}$ & $\begin{array}{l}\text { Male participants had blank, dull and sad expressions; females were } \\
\text { smiling and crying and displayed elated expressions; males were } \\
\text { seclusive and distracted, while females were cooperative and over } \\
\text { confident }\end{array}$ \\
\hline & & Posture \& psychomotor activity & Posture \& Mannerisms were slow, increased in some cases. \\
\hline \multirow{2}{*}{2.} & \multirow{2}{*}{ Speech } & Rate & $\begin{array}{l}\text { All participants began with push or pressured speech, later on the } \\
\text { rate of speech increased. }\end{array}$ \\
\hline & & Volume & $\begin{array}{l}\text { All participants spoke slowly, sometimes in whispers or muffled voices } \\
\text { and at other times were loud. }\end{array}$ \\
\hline \multirow{5}{*}{3.} & \multirow{5}{*}{ Emotional state: } & Mood & $\begin{array}{l}\text { 'Mood gets disturbed', 'Don't like anything', 'Get angry and hurt most } \\
\text { of the time', 'Can't control my anger', 'Don't feel like living'. }\end{array}$ \\
\hline & & Affect & $\begin{array}{l}\text { Males expressed less intense, constricted range of emotions during } \\
\text { the interview; they expressed sadness, guilt, remorse and lonely } \\
\text { feelings which were transient. }\end{array}$ \\
\hline & & Lability & $\begin{array}{l}\text { Females expressed intense emotions with full range and variety; they } \\
\text { showed elation, excitement, irritability, anger, fear and distrust; their } \\
\text { emotions were labile and unstable throughout the interview. }\end{array}$ \\
\hline & & Range: & $\begin{array}{l}\text { Affect of all participants were not compatible with the idea and } \\
\text { content of thought and with the description of the situation. }\end{array}$ \\
\hline & & $\begin{array}{l}\text { (Appropriateness to content and } \\
\text { situation) }\end{array}$ & \\
\hline \multirow{3}{*}{4.} & \multirow{3}{*}{ Thought processes } & Thought form & Loose associations and preoccupations in male participants. \\
\hline & & \multirow{2}{*}{ Thought content } & $\begin{array}{l}\text { Suicidal ideation in all participants; fears and somatic trends in male } \\
\text { participants. }\end{array}$ \\
\hline & & & $\begin{array}{l}\text { No sign of feelings of unreality or depersonalization, no illusions \& } \\
\text { hallucinations }\end{array}$ \\
\hline \multirow{4}{*}{5.} & \multirow{4}{*}{ Sensorium and mental capacity } & Orientation & Oriented to time, place \& person. \\
\hline & & Memory & $\begin{array}{l}\text { Remote memory was adequate; recent memory had minor flaws } \\
\text { while Immediate memory was poor. }\end{array}$ \\
\hline & & Attention and concentration & $\begin{array}{l}\text { Attention and concentration of all the participants were poor; males } \\
\text { were slow in paying attention while females were distracted. }\end{array}$ \\
\hline & & General intellectual evaluation & $\begin{array}{l}\text { General Intelligence involving general awareness, simple calculations } \\
\text { and abstract reasoning was average }\end{array}$ \\
\hline \multirow{3}{*}{6.} & \multirow{3}{*}{ Insight and judgment } & Health \& illness & $\begin{array}{l}\text { All participants had the knowledge and accepted that they have some } \\
\text { mental problem; they admitted that they cause trouble to others and } \\
\text { are different from others in their behaviour; they insisted that their } \\
\text { problems were not severe }\end{array}$ \\
\hline & & Need for treatment & $\begin{array}{l}\text { They expressed reluctance for treatment; although they wanted their } \\
\text { problems to be shared, heard and understood by someone. }\end{array}$ \\
\hline & & Plans \& goals & $\begin{array}{l}\text { They had no idea about their own plans, were dependent on family } \\
\text { members for any plan of action; they were uncertain about their goals. }\end{array}$ \\
\hline
\end{tabular}


The first section of MSE dealt with the general appearance of patients. This section did not provide any significant information. Facial expressions of males were dull, sad and reflected their feelings of loneliness while females were elated and were cooperative. Psychomotor activity was slow, increased in some cases. Speech of all clients was slow, they spoke in muffled voices and sometimes they were loud. As far as affect and emotional state was concerned, these patients conveyed rich information pertaining to the sudden shifts in emotional states which were not appropriate to the content expressed. Feelings of anger, hurt, boredom, and depression were common. Males showed unstable feelings of sadness, guilt, remorse and loneliness. Females showed intense and labile emotions like elation, excitement, irritability, anger, fear and distrust. Affect was not compatible with the idea and content of thoughts and situations. The strong and intense emotions or emotional cascades were observed during the interviews. In an emotional cascade, BPD individuals may experience a positive feedback loop in which the tendency to ruminate on negative emotional thoughts and feelings increases levels of negative emotions, and the increase in negative emotions, in turn, increases levels of attention to emotional stimuli, thus resulting in more rumination. The emotional cascade model of $\mathrm{BPD}^{37}$ suggests that the turbulent emotional experience of those with BPD is the result of emotional cascades which are vicious cycles of increasing rumination and negative emotions. This model attempts to provide a direct link between emotion dysregulation and behavioral dysregulation in BPD. This phenomenon may account for the extreme emotional experience observed in BPD, as well as why dysregulated behaviors are so central to BPD. Thought processes involved preoccupations, fears and somatic trends only in males. Suicidal ideation was common in all clients. Patients were extremely moody and their moodiness was associated with environmental precipitants, however minor or subtle. They not only changed from one mood state to another but the intensity of their feelings rapidly grew in severity. In fact, their affect reached such a level of intensity that it clouded their judgment or thought process and led to impulsive behaviors that were directly or indirectly self-destructive. The sensorium and mental capacity section showed that the remote memory of clients was adequate, recent memory was impaired while immediate memory was poor. They had poor attention and concentration as well as average general awareness, calculations and reasoning ability. Neuropsychological investigations suggest that $\mathrm{BPD}$ is associated with memory disturbances, including difficulties in the encoding and retrieval of complex multi-modal information. ${ }^{38-41}$ Insight and the judgment capacity of clients showed that they were aware of their mental problem (which was not severe) and that they caused trouble to others. They wanted their problems to be shared, heard and understood. They were uncertain about their plans and goals and were dependent on family members. Limited studies have been done using MSE for assessing BPD patients. One such study was by ${ }^{42}$ who have used MSE for assessing the emotional state of BPD patients. They used facial emotion recognition paradigms to investigate the bases of interpersonal impairments in BPD and found that an enhanced sensitivity to the mental states of others may be a basis for the social impairments in BPD. Evidence also suggests that BPD is mediated by disturbances within neural ${ }^{43,44}$ and neurochemical systems ${ }^{29-45}$ that support cognitive and emotional functions. The semi-structured interview schedule used in this study for seeking information about the patients is similar to Clinical Diagnostic Interview CDI. ${ }^{46-48}$ The CDI asks patients to provide detailed narratives about their symptoms, their school and work history, and their relationship history, focusing on specific examples of emotionally salient experiences.

\section{Conclusion}

The common clinical features of BPD patients as identified in case histories and MSE were anger, tension, anxiety, depression, sadness irritability, suicide attempts, associated disturbances in sleep, appetite and somatic symptoms and psychiatric illness among family members. Their moods were extremely changing, disturbed and uncontrollable. These symptoms resulted in poor performance, lack of interest in work or studies, poor memory and forgetfulness, frequent fights, few friends, isolation and abusing substances. Early home environment was either strict or permissive along with financial and marriage related worries. Patients were poor in studies, truants, stubborn, aggressive and were able to manipulate the situation during their childhood. They were lonely, avoided and punished by others. They had partial or no insight about their mental illness and were unclear and doubtful about things. Family attitudes of these patients revealed that family members were ignorant about the problem, they denied and concealed the problems from others and were reluctant to seek professional help. Stigma was another major barrier which labelled the client as 'mad' or having 'disturbed mind' leading them to reclusiveness or isolation. Societal attitudes were marked by shunning or avoiding the patient. They were not part of the mainstream resulting in social exclusion. There was denial, doubt and confusion on part of the patients regarding their own behavior (stigmatizing the self).

MSE Information revealed that clients" emotional state, mental capacity and insight \& judgment were basically impaired while speech \& thought processes were slightly affected. The most frequent symptoms characterizing BPD were anxiety, confusion \& doubt. Other common symptoms were anger, impulsive acts, splitting, guilt, loneliness, boredom, emptiness, dependency, uncertainty, attentional needs, counter dependency, intolerance of aloneness, substance abuse, and helplessness. Major areas of impairment or dysfunction included emotional states, cognitive functions, insight and judgment, interpersonal relationships and educational and vocational endeavors. A characteristic profile for evaluating emotional and cognitive functioning of BPD was developed in the study using case history and MSE. The common features, major symptoms and areas of impairment described the presentation and mode of expression of BPD features.

\section{Compliance with ethical standards}

Disclosure of potential conflicts of interest: This study was not funded by any agency. None of the authors received any funding. Hence there is no conflict of interest.

\section{Research involving human participants and/or animals}

Ethical approval: This article does not contain any studies with animals performed by any of the authors. All procedures performed in studies involving human participants were in accordance with the ethical standards of the institutional and/or national research committee or comparable ethical standards. Approval by the Institutional Ethics Committee of University of Allahabad, Allahabad for conducting research on human participants (Patients) was sought. An approval Letter was obtained which stated 'no objection' towards the conduction of the research by the investigator.

\section{Informed consent}

A consent letter was also signed by the participants before administering the tests to the participants. The consent letter provided information about the present research. This letter was an informed consent to the participants. Informed consent was obtained from all individual participants included in the study. 


\section{Acknowledgments}

None.

\section{Conflicts of interest}

Author declares there are no conflicts of interest.

\section{Funding}

None.

\section{References}

1. Coid J, Yang M, Tyrer P, et al. Prevalence and correlates of personality disorder in Great Britain. The Br J Psychiatry. 2006;188:423-431.

2. Crawford TN, Cohen P, Johnson JG. Self-reported personality disorder in the Children in the Community Sample: convergent validity and prospective validity in late adolescence and adulthood. J Pers Disord. 2005;19(1):30-52.

3. Lenzenweger MF, Lane MC, Loranger, et al. DSM-IV Personality Disorders in the National Comorbidity Survey Replication (NCS-R). Biol Psychiatry. 2007;62(6):553-564.

4. Grant BF, Chou SP, Goldstein RB, et al. Prevalence, correlates, disability, and comorbidity of DSM-IVborderline personality disorder: results from the Wave 2 National Epidemiologic Survey on Alcohol and Related Conditions. J Clin Psychiatry. 2008;69(4):533-545.

5. Zanarini MC, Frankenburg FR, Dubo ED, et al. Axis I Comorbidity of Borderline Personality Disorder. American Journal of Psychiatry. 1998;155(12):1733-1739.

6. Linehan M. Cognitive-behavioral treatment of borderline personality disorder. (1st edn), Guilford Press, USA. 1993. p.1-558.

7. Paris J. Childhood Adversity, Psychosocial Risk Factors, and the BPD Phenotype. 2006.

8. Figueroa E, Silk K. Biological Implications of Childhood Sexual Abuse in Borderline Personality Disorder. Journal of Personality Disorders. 1997;11(1):71-92.

9. Widiger TA, Frances, A, Warner L, et al. Diagnostic criteria for the borderline and schizotypal personality disorders. Journal of Abnormal Psychology. 1986;95(1):43-51.

10. Linehan, MM, Chiles JA, Egan KJ, et al. Presenting problems of parasuicides versus suicide ideators and nonsuicidal psychiatric patients. Journal of Consulting \& Clinical Psychology. 1986;54(6):880-881.

11. Petronis KR, Samuels JF, Moscicki EK, et al. An epidemiologic investigation of potential risk factors for suicide attempts. Soc Psychiatry Psychiatr Epidemiol. 1990;25(4):193-199.

12. Diekstra RFW, Van Egmond M. Suicide and attempted suicide in general practice: 1979-1986. Acta Psychiatric Scandinavia. 1989;79(3):268-275.

13. American Psychiatric Association. Diagnostic and statistical manual of mental disorders. (4th edn), American Psychiatric Association Publishing, USA. 2000.

14. American Psychiatric Association. Diagnostic and statistical manual of mental disorders (5th edn), American Psychiatric Association Publishing, USA. 2003.

15. Gardner DL, Leibenluft E, O'Leary KM, et al. Self-ratings of anger and hostility in borderline personality disorder. Journal of Nervous and Mental Disease. 1991;179(3):157-161.

16. Raine A. The psychopathology of crime: Criminal behavior as a clinical disorder. Academic Press Inc., USA. 1993
17. Russ MJ, Shearin EN, Clarkin JF, et al. Subtypes of selfinjurious borderline patients. American Journal of Psychiatry. 1993;150(12):1869-1871.

18. Linehan MM, Bohus M, Lynch TR. Dialectical behavior therapy for pervasive emotion dysregulation: theoretical and practical underpinnings. In: Gross JJ (Ed.), Handbook of emotion regulation. The Guilford Press, New York, USA. 2007. p.581-606.

19. Widiger TA, Trull TJ. Borderline and narcissistic personality disorders. In: Sutker PB \& Adams HE (Eds.), Comprehensive handbook of psychopathology. (2nd edn), Plenum Publishing Corp, USA. 1993. p.371-394.

20. Trull TJ, Sher KJ, Minks-Brown C, et al. Borderline personality disorder and substance use disorders: A review and integration. Clin Psychol Rev. 2000;20(2):235-253.

21. Links PS, Heslegrave RJ, Mitton JE, et al. Borderline psychopathology and recurrences of clinical disorders. Journal of Nervous and Mental Disease. 1995;183(9):582-586.

22. Oldham JM, Skodol AE, Kellman HD, et al. Comorbidity of axis I and axis II disorders. Am J Psychiatry. 1995;152(4):571-578.

23. Verheul R, Ball S, Van den Brink W. Substance abuse and personality disorders. In: Kranzler HR \& Rounsaville BJ (Eds.), Dual diagnosis and treatment: substance abuse and comorbid medical and psychiatric disorders. Marcel Dekker, USA. 1997. p.317-363.

24. Siever LJ, Davis KL. A psychobiologic perspective on the personality disorders, Am J Psychiatry. 1991;148:1647-1658.

25. Zanarini MC. Borderline personality disorder as an impulse spectrum disorder. In: Paris J (Ed.), Borderline Personality Disorder: Etiology and Treatment. American Psychiatric Publishing, Inc., USA. 1993. p.67-86.

26. Van Reekum R, Links PS, Fedorov C. Impulsivity in borderline personality disorder. In KR Silk (Ed.), Biological and neurobehavioural studies of borderline personality disorder. American Psychiatric Press, USA. 1994. p.1-22.

27. Links PS, Heslegrave R, Van Reekum R. Impulsivity: core aspect of borderline personality disorder. J Pers Disord. 1999;13(1):1-9.

28. Fyer MR, Frances AJ, Sullivan T, et al. Comorbidity of borderline personality disorder. Arch Gen Psychiatry. 1998;45(4):348-352.

29. Soloff PH, Lynch KG, Kelly TM, et al. Characteristics of suicide attempts of patients with major depressive episode and borderline personality disorder: a comparative study. Am J Psychiatry. 2000;157(4):601-608.

30. Nakao K, Gunderson JG, Phillips KA, et al. Functional impairment in personality disorders. Journal of Personality Disorders. 1992;6(1):24-33.

31. Bender DS, Dolan RT, Skodol AE, et al. Treatment utilization by patients with personality disorders. Ame J Psychiatry 158(2): 295-302.

32. Paris J (1996) Cultural factors in the emergence of borderline pathology. Psychiatry. 2001;59(2):185-192.

33. Pinto C, Dhavale HS, Nair S, et al. Borderline personality disorder exists in India. Journal of Nervous and Mental Disease. 2000;188(6):386-388.

34. Nath S, Patra DK, Biswas S, et al. Comparative study of personality disorder associated with deliberate self harm in two different age groups (15-24 years and 45-74 years): Indian Journal of Psychiatry. 2008;50(3):177-180.

35. Chopra HD, Beatson JA. Some Aspects of the Phenomenology of borderline Personality Disorder. Indian $J$ Psychiatry. 1988;30(3):221-226.

36. Choudhary S, Thapa K. Clinical Profile of Borderline Personality Disorder in North India. Psychological Studies. 2014;59(4):374-382. 
37. Selby EA, Anestis MD, Bender T, et al. An exploration of the emotional cascade model in borderline personality disorder. J Abnorm Psychol. 2009;118(2):375-387.

38. O $\gg$ Leary KM, Brouwers P, Gardner DL, et al. Neuropsychological testing of patients with borderline personality disorder. Am J Psychiatry. 1991;148(1):106-111.

39. Burgess JW. Neurocognitive impairment in dramatic personalities: histrionic, narcissistic, borderline, and antisocial disorders. Psychiatry Res. 1992;42(3):283-290.

40. Judd PH, Ruff RM. Neuropsychological dysfunction in borderline personality disorder. Journal of Personality Disorders. 1993;7(4):275-284.

41. Kurtz JE, Morey LC. Verbal memory dysfunction in depressed patients with and without borderline personality disorder. Journal of Psychopathology and Behavioural Assessment. 1999;21(2):141-156.

42. Fertuck EA, Jekal A, Song I, et al. Enhanced 'Reading the Mind in the Eyes' in borderline personality disorder compared to healthy controls. Psychol Med. 2009;39(12):1979-1988.
43. Driessen M, Herrman J, Stahl K, et al. Magnetic resonance imaging volumes of the hippocampus and the amygdala in women with borderline personality disorder and early traumatization. Arch Gen Psychiatry. 2000;57(12):1115-1122.

44. Donegan NH, Sanislow CA, Blumberg HP, et al. Amygdala hyperreactivity in borderline personality disorder: Implications for emotional dysregulation. Biol Psychiatry. 2003;54(11):1284-1293.

45. Hollander E, Stein DJ, DeCaria CM, et al. Serotonergic sensitivity in borderline personality disorder: preliminary findings. Am J Psychiatry. 1994;151(12):277-280.

46. Westen D, Muderrisoglu S. Reliability and validity of personality disorder assessment using a systematic clinical interview: Evaluating an alternative to structured interviews. Journal of Personality Disorders. $2003 ; 17: 350-368$.

47. Westen D, Muderrisoglu S. Clinical assessment of pathological personality traits. Am J Psychiatry. 2006;163(7):1285-1287.

48. Westen D, Muderrisoglu S, Fowler C, et al. Affect regulation and affective experience: Individual differences, group differences, and measurement using a Q-sort procedure. J Consult Clin Psychol. 1997;65(3):429-439. 